\title{
Oral tolerance in neonates: from basics to potential prevention of allergic disease
}

\author{
V Verhasselt ${ }^{1,2}$
}

Oral tolerance refers to the observation that prior feeding of an antigen induces local and systemic immune tolerance to that antigen. Physiologically, this process is probably of central importance for preventing inflammatory responses to the numerous dietary and microbial antigens present in the gut. Defective oral tolerance can lead to gut inflammatory disease, food allergies, and celiac disease. In the last two cases, the diseases develop early in life, stressing the necessity of understanding how oral tolerance is set up in neonates. This article reviews the parameters that have been outlined in adult animal models as necessary for tolerance induction and assesses whether these factors operate in neonates. In addition, we highlight the factors that are specific for this period of life and discuss how they could have an impact on oral tolerance. We pay particular attention to maternal influence on early oral tolerance induction through breast-feeding and outline the major parameters that could be modified to optimize tolerance induction in early life and possibly prevent allergic diseases.

\section{NEONATAL ORAL TOLERANCE INDUCTION IN ANIMAL MODELS IS POSSIBLE}

Studies in rodents have shown that oral tolerance ${ }^{1-3}$ may be more difficult to induce in neonates and infants than in adults. ${ }^{4}$ Thus, the administration of hen egg ovalbumin (OVA) to 1-day-old mice led to significant priming of an OVA-specific immune response in adulthood. In contrast, administration of OVA after the first week of age did induce tolerance. ${ }^{5}$ Similar results were obtained by Strobel and Ferguson, ${ }^{6}$ who showed that tolerance induction could be induced in mice after days 7-10 but not before. In addition, they showed that there was a temporary loss in the effectiveness of oral induction at the time of weaning. Defects in oral tolerance induction were also shown in neonatal pigs that developed hypersensitivity after weaning with preexposure to dietary proteins in the neonatal period. ${ }^{7}$ Similar defects were later observed in a rat model of experimental autoimmune encephalomyelitis; indeed, administration of myelin basic protein to rats induced tolerance when given after week 4 of life, while earlier administration resulted in priming and dysregulated experimental autoimmune encephalomyelitis. ${ }^{8}$ Additionally, these authors showed that systemic administration of myelin basic protein in neonatal rats could prevent this form of encephalomyelitis, showing that the defect in tolerance induction in neonates was specific to the oral route. In apparent contrast with that study, Maron et al. ${ }^{9}$ demonstrated that oral administration of insulin induced efficient tolerance as well as protection from type 1 diabetes in nonobese diabetic neonates.

Indirect oral antigen administration to neonatal mice through maternal milk has also been assessed for oral tolerance induction. Tolerance to OVA was induced by day 7, but the amounts of OVA required for tolerance to be induced in that time frame were about 1,000 times lower than those needed when the pups were directly gavaged. ${ }^{4}$ Other studies have shown that the breast-milk-mediated transfer of an antigen could prevent antigen-specific immune responses ${ }^{10-14}$ and allergic disease development in rodents. ${ }^{13}$ A physiological situation that illustrates very clearly the possibility of inducing tolerance to antigens present in breast milk is a child's exposure to cells and soluble human leukocyte antigen (HLA) molecules from the mother during pregnancy and through breast-feeding; antigens that are not transmitted genetically are called non-inherited maternal HLA antigens (NIMA). ${ }^{15}$ Elegant studies in rodents demonstrated that transfer of these HLA antigens from the mother to the pup in utero and through breast milk favor the acceptance of heart, skin, or bone marrow semi-allogeneic transplants that express NIMA. ${ }^{16,17}$ In the case of bone marrow transplant, the transfer of HLA antigen through breast milk was sufficient to prevent allogeneic reactions.

These studies suggest that, although neonatal oral tolerance induction is possible, the mechanisms involved are different

${ }^{1}$ INSERM, U924, Valbonne, France. ${ }^{2}$ Université de Nice-Sophia-Antipolis, Valbonne, France. Correspondence: V Verhasselt (verhasselt@ipmc.cnrs.fr)

Received 10 February 2010; accepted 8 April 2010; published online 19 May 2010. doi:10.1038/mi.2010.25 
from those in the adult. In the following paragraphs, we highlight the factors that are specific to this period of life and discuss how they could be modified to optimize oral tolerance induction in early infancy.

\section{MECHANISMS OF ORAL TOLERANCE INDUCTION IN NEONATES VS. ADULTS}

Oral tolerance in adults requires exposure to an optimal dose of an antigen by the oral route, the translocation of this antigen across the gut barrier, and its presentation by antigen-presenting cells to T lymphocytes, resulting in antigen-specific tolerance. In the following sections, we examine how these critical steps can be fulfilled in neonates.

\section{Do neonates encounter common environmental and dietary antigens by the oral route?}

The first question to be asked in determining whether oral tolerance can be induced in neonates and have a role in immune responses later in life is whether a neonate is exposed to environmental and dietary antigens by the oral route. According to the current World Health Organization (WHO) recommendation, infants receive milk from either the maternal breast or formula derived from cows during their first 6 months, after which solid food is progressively introduced. ${ }^{18,19}$ $\beta$-Lactoglobulin is a major antigen that is present in cow's milk in amounts of $\mathrm{mg} \mathrm{ml}^{-1}$ but is absent from human milk. Up to $3 \%$ of children fed cow's milk become allergic to this antigen. In addition to maternal-milk-specific proteins, breastfed children receive maternal dietary antigens. ${ }^{20}$ Studies in humans have documented the presence of antigenically intact food proteins in human milk, including bovine $\beta$-lactoglobulin, hen egg OVA, gliadin from wheat, and Ara $\mathrm{H} 1$ from peanuts. $^{20}$ These antigens are found in concentrations ranging from 100 to $400 \mathrm{ng} \mathrm{ml}^{-1}$, with the concentrations varying markedly from one individual to another. We observed in a mouse model that airborne antigens could also be found in breast milk. ${ }^{13}$ Although surprising when first considered, this observation is easily explained because $95 \%$ of inhaled antigens are found in the gut. ${ }^{21,22}$ Thus, oral exposure to dietary and environmental antigens occurs early in life, but both the nature and the amount of the antigens encountered vary according to the mode of feeding: whereas formula-fed children receive large amounts of cow's-milk antigens exclusively, children who are breast-fed receive daily, from birth until weaning, minute amounts of numerous antigens ingested by the mother.

\section{How are antigens handled in the neonatal gut?}

Before reaching her milk, antigens ingested by a mother are processed in the digestive tract, and this process may have consequences for the generation of tolerogenic forms of an antigen. ${ }^{23,24}$ Studies of oral tolerance in adult rodents have demonstrated that antigen handling in the gut is necessary to confer tolerogenic properties to orally administered antigens. ${ }^{23,24}$ Among the gut's factors involved in the generation of tolerogenic forms of antigens are the low stomach
$\mathrm{pH}^{25}$ and digestion by pancreatic enzymes. ${ }^{26}$ The gastric $\mathrm{pH}$ of young infants ranges from 3 to 5 , compared with 1-2 in adults, ${ }^{27}$ and secretion of pancreatic enzymes is low at birth and increases during infancy. ${ }^{27}$ Therefore, the processing of a dietary antigen in the maternal digestive tract before it reaches the milk may favor the induction of antigen-specific tolerance in the neonate. In support of this hypothesis, we found that, although both intravenous and oral administration of an antigen to a lactating mother result in its appearance in breast milk, only oral administration to the mother is able to induce tolerance in the breast-fed mice (V Verhasselt, unpublished data).

The amount of antigen that will be able to cross the gut barrier is influenced by the presence in the gut lumen of antigen-specific immunoglobulins (Ig).$^{3,28,29}$ In adults, secretory IgA represents the predominant antibody class in intestinal secretions. ${ }^{3,28,29}$ This class of noninflammatory Ig inhibits the adherence of bacteria and viruses to mucosal surfaces and microbial colonization. IgA can also trap food antigens; this is responsible for immune exclusion of dietary antigens and favors their degradation by pancreatic enzymes. In addition, the antigens that have been translocated through the epithelial barrier induce the synthesis of antigen-specific secretory IgA, and antigens bound to IgA are then actively transported from the lamina propria back to the lumen. ${ }^{30}$ IgA may also exert an immunoregulatory effect. ${ }^{31-34}$ In contrast to animal studies showing that oral tolerance can be induced in adult mice in the absence of secretory IgA, ${ }^{35}$ epidemiological studies suggest that IgA-deficient individuals are more susceptible to various allergies, including food allergies. ${ }^{36}$ Neonates are deficient in IgA synthesis, but maternal IgA in breast milk can substitute efficiently for this lack of endogenous synthesis. ${ }^{37}$ Maternal dietary antigens are commonly found bound to IgA in milk. ${ }^{38,39}$ Studies have shown an inverse correlation between the levels of IgA in breast milk and further allergy development in breast-fed children, supporting a possible role of IgA in oral tolerance. ${ }^{40-42}$ However, maternal IgA in breast milk was not necessary for tolerance induction in neonates through breast milk in an animal model of antigen transmission. ${ }^{13,43}$ Antigens in breast milk may also be found associated with maternal IgG. In an animal model, we noted that antigen-sensitized mothers that are exposed to the antigen during lactation present high level of IgG-antigen immune complexes in breast milk. ${ }^{43}$ In addition, and as discussed later, the presence of IgG-antigen immune complexes in breast milk was associated with the induction of a much more profound and long-lasting antigen-specific tolerance in breast-fed mice as compared with tolerance induced when the antigen was found free in breast milk. ${ }^{43}$ In humans, dietary or environmental antigen-IgG immune complexes might also be found in breast milk. Indeed, such immune complexes were found in sera of both healthy and atopic individuals, ${ }^{44-46}$ and a receptor specialized in IgG or IgG-immune complex transfer, the FcRn (see below), is expressed in the epithelium of mammary gland, allowing the transfer of IgG-IgG immune complexes from sera to the milk. ${ }^{47,48}$ 


\section{How are antigens transferred across the gut barrier and presented to the immune system in neonates?}

Intestinal barrier. There are marked differences in the timing and extent of intestinal maturation in various animal species, and the variations reflect the duration of the gestational period. In mice and rats, which are born after a short gestation, the intestine is poorly developed at birth, whereas in species that have a long gestation period-e.g., guinea pig, sheep, and pig-intestinal development occurs earlier in utero. These species are more representative of human development in the fetal and neonatal periods. ${ }^{49}$ The gut maturation process is associated with a decrease in intestinal permeability and a consequent decrease in the transport of macromolecules across the intestinal barrier. This phenomenon, called gut closure, takes place in rodents at the time of weaning and within the first postnatal days in humans and other species with a long gestational period. Maternal colostrum and milk contain gut epithelium growth factors, such as epidermal growth factor and transforming growth factor, that stimulate intestinal growth and development, accelerate gut closure, and might affect antigen transfer. ${ }^{50}$ Some studies suggest that alterations in gut permeability might be involved in the pathogenesis of gut inflammatory disease. ${ }^{51}$ For example, permeability was increased in an animal model of food allergy and in humans sensitive to cow's milk after milk provocation. ${ }^{52}$ It is not clear whether gut permeability influences induction of oral tolerance, but increased permeability in conjunction with other activating factors of the immune system, such as gut infection, might favor allergy development. ${ }^{28}$

Neonatal Fc receptor. Although passive transcellular transport of proteins leads to degradation of more than $90 \%$, there also exists active protected transport across the epithelial cells. ${ }^{53,54}$ One well-described protected transport is that of IgG. Mice and rats receive maternal IgG mainly in the postnatal period through milk, whereas other species_-including humansreceive maternal passive immunity both before birth (by transplacental transfer of IgG) and after birth (through milk). The existence of a receptor involved both in IgG transfer across the placenta and gut and in the regulation of serum IgG was proposed by Brambell ${ }^{55}$ in the 1960s and was finally identified as the neonatal Fc receptor in the 1990s. ${ }^{56-58}$ This receptor, which is composed of a transmembrane protein associated with $\beta-2$ microglobulin, is structurally related to the major histocompatibility complex class I molecule. In the gut, it is expressed in the proximal small intestine until weaning in rodents and throughout life in humans. The crucial role of this receptor for humoral immunity of the neonate was identified when neonatal mice deficient in the FcRn heavy chain proved to be unable to absorb IgG from maternal milk. ${ }^{56-58}$ This receptor may also be important for antigen transport given that it allows antigen bound to IgG to cross the intestinal barrier without being degraded. This was very elegantly demonstrated 30 years ago by Abrahamson and colleagues, ${ }^{59}$ who described the transfer of an antigen by gut epithelial cells in neonatal rats and observed that detectable amounts could be found in the lamina propria only when the antigen was bound to antigen-specific IgG. Using a human FcRn transgenic construct, Blumberg and colleagues showed that antigen-IgG complexes were transcytosed across the lamina propria in adult mice and transferred to lamina propria dendritic cells (DCs), resulting in an effector immune response. ${ }^{60,61}$ We found that antigen-IgG immune complexes were present in mouse breast milk on administration of an antigen to lactating antigen-sensitized mothers. Milk-borne antigen-IgG immune complexes were efficiently transported across the intestinal barrier using the FcRn. This transfer resulted in induction of regulatory T cells (Tregs) and a much more potent tolerance induction and protection from allergic airway disease as compared with those induced by the transfer of free antigen. ${ }^{43}$ Such an efficient tolerance induction probably relies on the protected antigen transfer across the gut barrier when antigen is complexed to IgG by the use of the FcRn. This demonstrates in a physiological model the role of this receptor in oral tolerance induction in neonates.

Antigen capture and presentation by DCs. In addition to epithelial cell transport, antigen uptake by $M$ cells in Peyer's patches and by DCs expanding dendrites between epithelial cells allows the transfer of intact antigen across the intestinal barrier in adult rodents. ${ }^{2,62}$ However, their direct role in oral tolerance induction is uncertain. Where tolerance induction takes place is a matter of debate, but the best evidence suggests that mesenteric lymph nodes have a crucial role. ${ }^{2,62,63}$ Peyer's patches and mesenteric lymph nodes are already present at birth in both rodents and humans, ${ }^{64}$ and microbial microbiota and diet affect their development (see below). In addition to antigen uptake, some DC subpopulations are specialized in tolerance induction in the gut. In particular, one subpopulation expressing CD103 has a key role in the induction of Tregs in mesenteric lymph nodes through the local production of retinoic acid. ${ }^{65,66}$ The absolute number of DCs is reduced by several logs in the neonate body as compared with adults. There are also differences in the representation of various subpopulations, such as plasmacytoid DCs, that are more numerous in neonates as well as in their function, such as IL-12 secretion, which is reduced in neonates. ${ }^{67}$ Few reports describe the presence of DCs in gut mucosa and gut-associated lymphoid tissues in neonates. Thus, major histocompatibility complex class II-positive cells with DC morphology have been identified in neonatal lamina propria and the mesenteric lymph nodes. ${ }^{68,69}$ However, where oral tolerance induction takes place in the neonate and which cells are involved in this process need clarification.

\section{Is the neonatal immune system prone to tolerance induction upon antigen presentation of orally administered antigen?}

Once an antigen has crossed the intestinal barrier, tolerance induction requires its presentation to lymphocytes followed by their deletion or the induction of Tregs. Pioneer studies by Medawar suggested that the neonatal immune system is immunologically immature and prone to tolerance induction. ${ }^{70-72}$ This was shown in a mouse model in which systemic administration of an alloantigen in utero or in the perinatal period resulted in tolerance in adulthood to a skin graft expressing 
the same alloantigen, whereas the same protocol in adult led to accelerated graft rejection. ${ }^{70,71}$ Later studies showed not only that neonates are biased for $\mathrm{T}$ helper type 2 cell responses as compared with adults but also that any kind of immune response could be induced in neonates under appropriate conditions. ${ }^{67,73-}$ 75 Thus, by modifying the dose of antigen, the adjuvant used, or the ratio of antigen-presenting cells to responder cells, adult-like effector immune responses could be induced in neonates. The concept of immune system immaturity in neonates has been established on the basis of rodent studies. Compared with data obtained from humans, it appears that mice are immunologically less mature at birth than humans, and it has been proposed that 7-day-old mice are most comparable to human newborns. ${ }^{67}$ Nevertheless, a process of immune maturation is also observed in humans, as illustrated by the observation that many food allergies, such as cow's milk allergy in early infancy, have a high spontaneous remission rate at around 3 years of age, most likely due to a progressive shift from Thelper type 2 cells' immune response to Treg induction. ${ }^{76}$

Although genetic factors are important risk factors for defective tolerance induction and allergy development, ${ }^{77-79}$ they cannot be modified. However, there is compelling evidence that modifiable factors such as gut bacterial colonization and diet have a key role in the maturation of the neonatal immune system and its further susceptibility to tolerance induction.

Gut microbiota and immune system maturation. The gastrointestinal tract of the newborn is sterile, and it is colonized soon after birth by numerous types of microorganisms. Colonization is complete after approximately 1 week, but the number and species of bacteria fluctuate markedly during the first year. Once established, the microbiota are surprisingly stable, and they are specific to each individual. ${ }^{80-82}$ The impact of the gut microbiota on immune system maturation was demonstrated by major alterations of the immune system in germ-free mice: hypoplastic Peyer's patches with few germinal centers and greatly reduced numbers of IgA-producing plasma cells and lamina propria $\mathrm{CD} 4{ }^{+} \mathrm{T}$ cells. In addition, the immunological abnormalities in germ-free animals are not confined to the mucosal immune system; spleen and lymph nodes are relatively structureless, with abnormal B- and T-cell zones. These morphological features are associated with important functional changes, such as hypogammaglobulinemia, T helper type 2 cell shift, and defects in oral tolerance induction. ${ }^{82-84}$ Perhaps surprisingly, colonization of germ-free mice by a single, unique species of bacteria-e.g., Bacteroides fragilis ${ }^{85}$ or segmentous filamentous bacteria, ${ }^{86}$ - was shown to be sufficient to restore the development of a multifaceted adaptive immune response. ${ }^{87}$ However, qualitative modification of gut microbiota affects immune response development and efficiency of tolerance induction. Thus, in animals, certain microbiota have been shown to protect from autoimmune diabetes development. ${ }^{88}$ In humans as well, differences in the composition of gut microbiota have been reported among healthy infants both in countries with a low prevalence of allergies and in those with a high prevalence, and among allergic and nonallergic infants in both environments. ${ }^{82}$
A similar phenomenon has been reported for autoimmune diseases ${ }^{89}$ suggesting that qualitative alteration of the microbiota could affect the efficacy of tolerance vs. sensitization upon antigen encounter. However, exact identification of particular protective or potentially harmful bacteria species has not yet been achieved. Probably the most convincing effect of a protective microbiota is found in the presence of Bifidobacterium and Lactobacillus species in the gut microbiota. In line with this is the observation that the administration of probiotics of these bacteria species had encouraging results for allergy prevention in infants, although more studies are needed to firmly establish their protective role. ${ }^{82}$ Factors in the development of a particular microbiota include the maternal microbiota, the delivery method, incidental microbial encounters, and the child's diet. ${ }^{80-82}$ In particular, breast milk contains oligosaccharides and glycoproteins that promote the growth of health-promoting bacteria. Finally, and perhaps intriguingly, bacteria from the mother's flora were found in the milk of mice and humans. ${ }^{90}$

Diet and immune system maturation. Although much attention has been paid to the effects of bacterial stimuli on immune system development, gut microbiota are prominent in the large intestine, whereas most of the gut's lymphoid immune system is located in the small intestine, where protein absorption predominantly takes place. Antigenic constituents of food clearly exert a stimulatory effect on the neonatal immune system, as mice fed hydrolyzed milk proteins or fed parenterally have fewer $\mathrm{B}$ and $\mathrm{T}$ cells. Moreover, parenterally fed babies have reduced gut immune system development. ${ }^{28}$ The effect of food antigens on development of the immune system has been studied in detail by Faria and colleagues. ${ }^{91}$ These authors observed that mice receiving a balanced amino-acid-based protein-free diet had poorly developed gut-associated lymphoid tissue and displayed low numbers of lamina propria cells and intraepithelial lymphocytes as well as signs of decreased systemic immunity. Fariass group also showed that nasal tolerance was impaired in those mice. ${ }^{92}$ Thus, whereas gut colonization might be important for immune system maturation before weaning, diet might be just an as important factor after weaning. However, diet may affect immune system development even before weaning, because the food antigen load in breast-fed vs. formula-fed children is very different. Finally, as discussed above, in addition to its direct antigenic stimulatory effect, diet can influence the immune system by introducing factors that influence microbiota development.

Local mucosal factor for tolerance induction. Transforming growth factor- $\beta$ (TGF- $\beta$ ) is a key cytokine for immune tolerance homeostasis, as illustrated by the overwhelming and deadly systemic inflammation in mice deficient in TGF- $\beta 1$. TGF- $\beta$ seems to have a key role in the induction of different subclasses of Tregs involved in oral tolerance, such as those producing IL-10 (Tr1) or expressing FoxP3.${ }^{93}$ It is also recognized as an important effector molecule that defines orally induced regulatory $\mathrm{T}$ helper type 3 cells. ${ }^{3}$ Although hematopoietic cells produce it, the local source of TGF- $\beta$ seems to be the epithelial cells. ${ }^{1,66}$ Endogenous levels of TGF- $\beta$ in the intestine are low at birth and 
increase toward weaning, whereas maternal milk is rich in TGF$\beta .^{94}$ TGF- $\beta$ in maternal milk is sufficient to rescue TGF- $\beta$-deficient mice from widespread inflammation. ${ }^{95}$ In addition, elegant studies by Penttila et al. demonstrated that rats fed formula milk without TGF- $\beta$ displayed intestinal inflammation ${ }^{96}$ and developed an allergic response to bovine $\beta$-lactoglobulin when on a susceptible background. ${ }^{97}$ Supplementation of artificial milk with physiological amounts of TGF- $\beta$ was sufficient to prevent intestinal inflammation and immune response to $\beta$-lactoglobulin. In agreement with these studies, breast-feeding-induced tolerance to a soluble antigen transferred from the mother to the neonate through breast milk required the presence of TGF- $\beta$ in milk. ${ }^{13}$ However, TGF- $\beta$ was not required when the antigen was associated with IgG in milk, suggesting that this suppressive factor is necessary only under suboptimal conditions of antigen transfer to the mice. ${ }^{43}$ TGF- $\beta$ may also be indirectly involved in tolerance induction given that milk TGF- $\beta$ exerts its effect on epithelial cells during lactation and prevents epithelium activation while enhancing gut barrier function. ${ }^{50,98}$ As a matter of fact, TGF- $\beta$ content in milk was shown to correlate inversely with the risk of allergy development in breast-fed children. ${ }^{99}$

IL-10 is another suppressive cytokine that has a key role in the mechanisms of gut tolerance. ${ }^{3}$ Interestingly, IL-10 is produced by B-1 cells, a subpopulation of B cells that is present in much higher proportions in neonates than in adults-40\% in neonates vs. $1 \%$ in adults - which suggests that this population may have a role in oral tolerance in neonates. ${ }^{100}$ In a model of breast-milkmediated antigen transfer, we found that neither IL-10 in milk ${ }^{13}$ nor IL-10 produced by the neonate was required for induction of oral tolerance (Mosconi and Vevhasselt unpublished data).

In addition to TGF- $\beta$ and IL-10, much attention has been paid in recent years to retinoic acid and its precursor vitamin $A$, and its role in the generation of Tregs in the gut. Thus, gut DCs expressing the CD103 molecule are able to convert vitamin $\mathrm{A}$ into retinoic acid and are specialized in the induction of gut-homing FoxP3 ${ }^{+}$Tregs. ${ }^{65,66}$ Retinoic acid was shown to promote the expression of the gut-homing receptor CCR9 and $\alpha 4 \beta 7$ integrin on the interacting lymphocytes and to act in synergy with TGF- $\beta$ on the induction of FoxP ${ }^{+}$Tregs. In addition, retinoic acid favors class switching to IgA. Thus, the availability of food-derived vitamin $\mathrm{A}$ is a unique aspect of the gut mucosal milieu that probably has a major influence on tolerance induction toward orally administered antigen. Vitamin $A$ is present in both breast milk and formula milk. In poorly developed countries, lactating mothers and infants who benefit from vitamin A supplementation not only are protected from the most severe complication of vitamin A deficiency-night blindness (xerophtalmie) - but also show less morbidity and mortality due to infections. ${ }^{101,102}$ The impact of maternal milk or formula-derived vitamin A on oral tolerance induction in neonates and infant has not yet been assessed.

The mechanisms put forward in the different settings of oral tolerance in neonates need to be further analyzed, but they seem to involve anergy or deletion, ${ }^{9,103}$ a defect in T helper cell function, but no Treg induction ${ }^{10}$ or the induction of regulatory $\mathrm{CD} 4{ }^{+} \mathrm{CD} 25^{+}$Tregs in the case of breast milk transfer of non-inherited maternal HLA antigens ${ }^{17}$ or IgG-complexed antigen. ${ }^{43}$

\section{ORAL TOLERANCE INDUCTION IN EARLY LIFE: A STRATEGY TO PREVENT ALLERGIC DISEASE?}

In adults, allergy prevention is classically based on allergen avoidance. This approach has been extended to fetuses, neonates, and infants by promoting the avoidance of allergen exposure during pregnancy, lactation, and the first years of life. However, these recommendations have not yielded the expected resultsthe prevalence of food allergy has continued to rise in recent years, and prospective studies assessing allergen avoidance have failed to show a significant long-term reduction in food allergies. ${ }^{18,20,104-107}$ Recently, the concept of oral tolerance to dietary antigens in neonates and infancy has emerged, leading some to propose the opposite approach: deliberate exposure to dietary and environmental allergens to induce tolerance and prevent allergic disease. ${ }^{18,77,106}$ This approach is supported by animal studies and by prospective studies that have shown that exposure to cereals before 6 months of age decreases the incidence of wheat allergy as compared with later introduction. ${ }^{108}$ Also, studies on the incidence of peanut allergy showed that it is found less frequently in places where peanuts are consumed throughout pregnancy and early childhood than it is in Western industrialized societies, where peanuts are usually avoided in pregnancy and infancy. ${ }^{77,109}$ Interestingly, in contrast to early oral exposure, cutaneous early antigen exposure favors sensitization rather than tolerance induction, highlighting the importance of the route of early antigen exposure in the subsequent development of immune responses. ${ }^{77}$ Ongoing studies are assessing the impact of early (4-11 months) peanut introduction into the diet of highrisk infants on peanut allergy development. ${ }^{18,77,106}$

As discussed above, animal studies suggest that breast-feeding enhances oral tolerance induction in neonates. In this respect, reports of allergy to dietary antigens transmitted through milk, such as cow's-milk allergy in exclusively breast-fed children, might be surprising. However, cow's-milk allergy is much less frequent in breast-fed children than in bottle-fed children $(0.5$ vs. $3 \%$, respectively), ${ }^{110}$ and most epidemiological studies show that breast-fed children develop fewer allergic diseases than bottle-fed children. ${ }^{105,107,111,112}$ The protective effects of breastfeeding on allergic disease development in humans might be due to the lower incidence in breast-fed children of infections such as respiratory syncytial virus that are known to predispose to development of asthma. In addition, as discussed above, we believe that breast-feeding might be protective because of both the presence of numerous allergens in breast milk that are absent from artificial milks and their tolerogenic presentation due to breastfeeding related factors such as antigen handling by maternal gut, allergens found in immune complexes in milk, the presence of tolerogenic immune mediators in milk, increased gut maturation and a microbiota favoring tolerance induction in breast-fed children (Figure 1). Variability in allergen and tolerogenic factor levels in milk might contribute to the controversy over the prevention of allergic disease via breastfeeding. ${ }^{105,107,111,112}$ In addition, as discussed above, our recent 


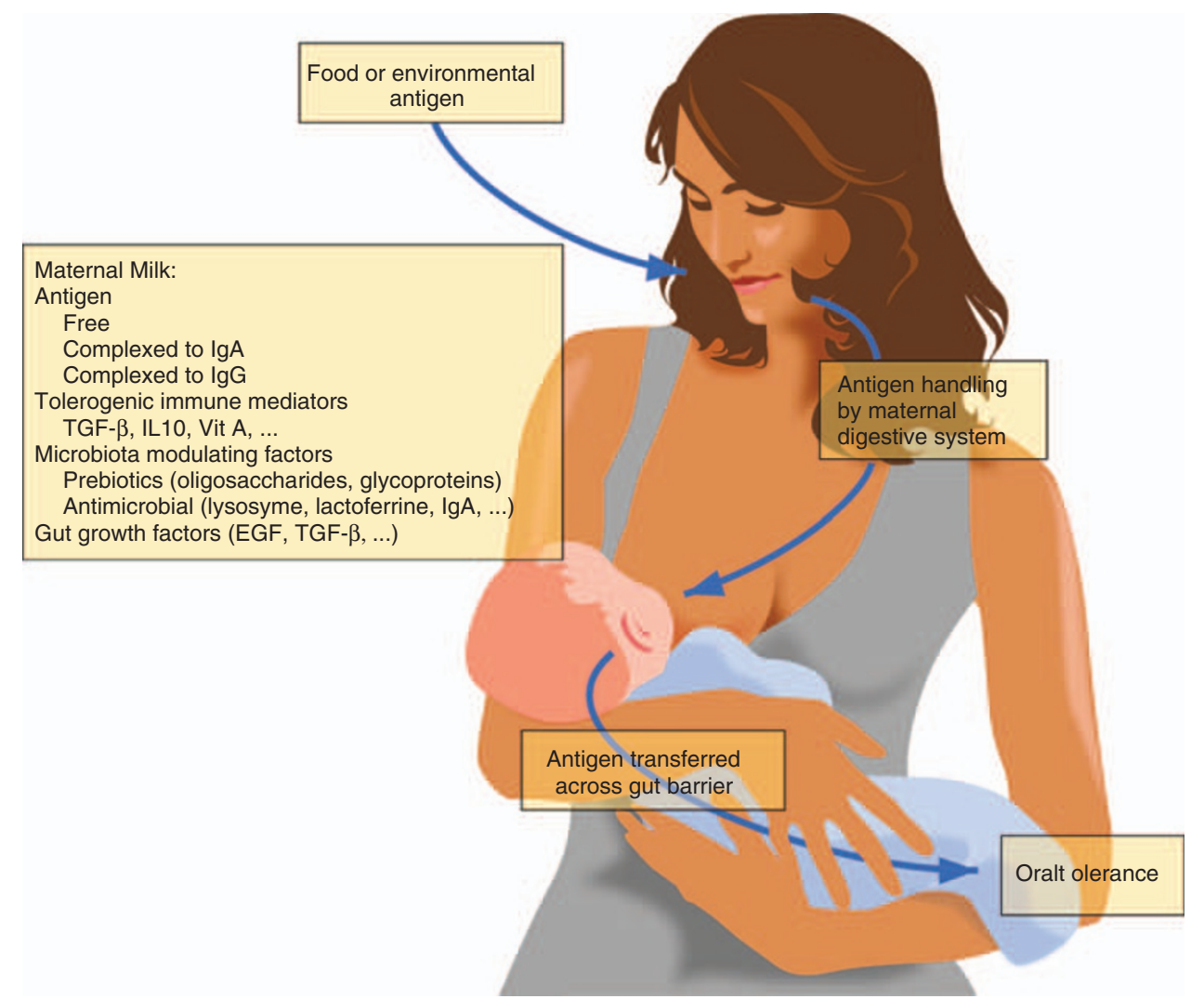

Figure 1 Possible maternal influence on neonatal tolerance induction through breast-feeding. Before reaching the milk, ingested airborne and dietary antigens are handled by the maternal digestive system, which could contribute to the generation of tolerogenic peptides. Depending on the maternal antigen exposure and mammary gland permeability, various amounts of antigen will be found in breast milk. Maternal sensitization to the ingested allergens will dictate whether the transferred antigens will be found in the milk free or complexed to antigen-specific IgA and IgG. The presence of IgA will trap antigens and prevent their transfer to the child, whereas antigen bound to IgG will be very efficiently transferred across the gut barrier using the FCRn. Gut epithelium maturation will be accelerated by the presence of growth factors such as epidermal growth factor (EGF) and transforming growth factor (TGF) in maternal milk. The presence of immunomodulating factors in milk such as TGF- $\beta$ will favor tolerance induction to the transferred antigens. Finally, prebiotics, such as oligosaccharides, that are present in breast milk will lead to the development of a microbiota promoting immune tolerance induction.

observations suggest that maternal sensitization to dietary and environmental antigens will profoundly affect the efficiency of tolerance induction through breast-feeding. ${ }^{43}$ In this regard, it is interesting to note that a meta-analysis of epidemiological studies on breast-feeding protection from allergic disease concluded that protection is particularly likely in the presence of a family history of atopy. ${ }^{112}$

\section{CONCLUDING REMARKS}

Immune responses induced in early life to environmental and dietary antigens will be decisive for children and their adult response to these antigens, and they will condition development of immune-mediated diseases such as allergies and autoimmunity. Maternal influence on neonatal tolerance induction through breast-feeding is probably of great importance because of dietary and environmental antigen transfer through breast milk and the pleiotropic effects of breast-feeding on gut and immune system maturation. In addition, maternal history and maternal sensitization to common environmental and food antigens will probably affect antigen transfer to the breastfed child along with tolerance induction.
Strategies aimed at improving oral tolerance in neonates will need to take into account the neonate's intrinsic characteristics and maternal influence through breast-feeding.

\section{ACKNOWLEDGMENTS}

I thank Eric Mosconi, Nicolas Glaichenhaus, Ana Faria, Momtchillo Russo, and Henry Verhasselt for critical reading of this article and Frank Aguila for figure design. This work was supported by grants from the Agence Nationale de la Recherche (SEST).

\section{DISCLOSURE}

The author declares no conflict of interest.

(C) 2010 Society for Mucosal Immunology

\section{REFERENCES}

1. Smith, K.M., Eaton, A.D., Finlayson, L.M. \& Garside, P. Oral tolerance. Am. J. Respir. Crit. Care Med. 162, S175-S178 (2000).

2. Mowat, A.M. Anatomical basis of tolerance and immunity to intestinal antigens. Nat. Rev. Immunol. 3, 331-341 (2003).

3. Faria, A.M. \& Weiner, H.L. Oral tolerance. Immunol. Rev. 206, 232-259 (2005).

4. Strobel, S. Neonatal oral tolerance. Ann. NY Acad. Sci. 778, 88-102 (1996). 
5. Hanson, D.G. Ontogeny of orally induced tolerance to soluble proteins in mice. I. Priming and tolerance in newborns. J. Immunol. 127, 1518-1524 (1981).

6. Strobel, S. \& Ferguson, A. Immune responses to fed protein antigens in mice. 3. Systemic tolerance or priming is related to age at which antigen is first encountered. Pediatr. Res. 18, 588-594 (1984).

7. Miller, B.G. et al. The importance of dietary antigen in the cause of postweaning diarrhea in pigs. Am. J. Vet. Res. 45, 1730-1733 (1984).

8. Miller, A., Lider, O., Abramsky, O. \& Weiner, H.L. Orally administered myelin basic protein in neonates primes for immune responses and enhances experimental autoimmune encephalomyelitis in adult animals. Eur. J. Immunol. 24, 1026-1032 (1994).

9. Maron, R., Guerau-de-Arellano, M., Zhang, X. \& Weiner, H.L. Oral administration of insulin to neonates suppresses spontaneous and cyclophosphamide induced diabetes in the NOD mouse. J. Autoimmun. 16, 21-28 (2001).

10. Komatsu, T., Okao, M., Miyamoto, H., Chen, T. \& Shinka, S. Effects of early antigen exposure through lactation on later specific antibody responses in mice. J. Immunol. 141, 2895-2906 (1988).

11. Telemo, E., Jakobsson, I., Westrom, B.R. \& Folkesson, H. Maternal dietary antigens and the immune response in the offspring of the guinea-pig. Immunology 62, 35-38 (1987).

12. Korotkova, M., Telemo, E., Yamashiro, Y., Hanson, L.A. \& Strandvik, B. The ratio of $n-6$ to $n-3$ fatty acids in maternal diet influences the induction of neonatal immunological tolerance to ovalbumin. Clin. Exp. Immunol. 137, 237-244 (2004).

13. Verhasselt, V. et al. Breast milk-mediated transfer of an antigen induces tolerance and protection from allergic asthma. Nat. Med. 14, 170-175 (2008).

14. Auerbach, R. \& Clark, S. Immunological tolerance: transmission from mother to offspring. Science 189, 811-813 (1975).

15. van Rood, J.J. \& Claas, F. Both self and non-inherited maternal HLA antigens influence the immune response. Immunol. Today 21, 269-273 (2000).

16. Andrassy, J. et al. Tolerance to noninherited maternal $\mathrm{MHC}$ antigens in mice. J. Immunol. 171, 5554-5561 (2003).

17. Aoyama, K. et al. Improved outcome of allogeneic bone marrow transplantation due to breastfeeding-induced tolerance to maternal antigens. Blood 113, 1829-1833 (2009).

18. Prescott, S.L. et al. The importance of early complementary feeding in the development of oral tolerance: concerns and controversies. Pediatr. Allergy Immunol. 19, 375-380 (2008).

19. Host, A. et al. Dietary prevention of allergic diseases in infants and small children. Pediatr. Allergy Immunol. 19, 1-4 (2008).

20. Palmer, D.J. \& Makrides, M. Diet of lactating women and allergic reactions in their infants. Curr. Opin. Clin. Nutr. Metab. Care 9, 284-288 (2006).

21. Willoughby, J.B. \& Willoughby, W.F. In vivo responses to inhaled proteins. I. Quantitative analysis of antigen uptake, fate, and immunogenicity in a rabbit model system. J. Immunol. 119, 2137-2146 (1977).

22. Holt, P.G., Batty, J.E. \& Turner, K.J. Inhibition of specific IgE responses in mice by pre-exposure to inhaled antigen. Immunology 42, 409-417 (1981).

23. Bruce, M.G. \& Ferguson, A. The influence of intestinal processing on the immunogenicity and molecular size of absorbed, circulating ovalbumin in mice. Immunology 59, 295-300 (1986).

24. Peng, H.J., Turner, M.W. \& Strobel, S. The generation of a "tolerogen" after the ingestion of ovalbumin is time-dependent and unrelated to serum levels of immunoreactive antigen. Clin. Exp. Immunol. 81, 510-515 (1990).

25. Untersmayr, E. et al. Antacid medication inhibits digestion of dietary proteins and causes food allergy: a fish allergy model in BALB/c mice. J. Allergy Clin. Immunol. 112, 616-623 (2003).

26. Michael, J.G. The role of digestive enzymes in orally induced immune tolerance. Immunol. Invest. 18, 1049-1054 (1989).

27. Agunod, M., Yamaguchi, N., Lopez, R., Luhby, A.L. \& Glass, G.B. Correlative study of hydrochloric acid, pepsin, and intrinsic factor secretion in newborns and infants. Am. J. Dig. Dis. 14, 400-414 (1969).

28. Brandtzaeg, P.E. Current understanding of gastrointestinal immunoregulation and its relation to food allergy. Ann. NY Acad. Sci. 964, 13-45 (2002).

29. Levinsky, R.J. Factors influencing intestinal uptake of food antigens. Proc. Nutr. Soc. 44, 81-86 (1985).

30. Robinson, J.K., Blanchard, T.G., Levine, A.D., Emancipator, S.N. \& Lamm, M.E. A mucosal IgA-mediated excretory immune system in vivo. J. Immunol. 166, 3688-3692 (2001).
31. Smits, H.H. et al. Cholera toxin B suppresses allergic inflammation through induction of secretory IgA. Mucos. Immunol. 2, 331-339 (2009).

32. Favre, L., Spertini, F. \& Corthesy, B. Secretory IgA possesses intrinsic modulatory properties stimulating mucosal and systemic immune responses. J. Immunol. 175, 2793-2800 (2005).

33. Sletten, G.B., Halvorsen, R., Egaas, E. \& Halstensen, T.S. Caseinspecific immunoglobulins in cow's milk allergic patient subgroups reveal a shift to IgA dominance in tolerant patients. Pediatr. Allergy Immunol. 18, 71-80 (2007).

34. Pilette, C., Durham, S.R., Vaerman, J.P. \& Sibille, Y. Mucosal immunity in asthma and chronic obstructive pulmonary disease: a role for immunoglobulin A? Proc. Am. Thorac. Soc. 1, 125-135 (2004).

35. Karlsson, M.R., Johansen, F.E., Kahu, H., Macpherson, A. \& Brandtzaeg, P. Hypersensitivity and oral tolerance in the absence of a secretory immune system. Allergy 65, 561-570 (2010).

36. Janzi, M. et al. Selective IgA deficiency in early life: association to infections and allergic diseases during childhood. Clin. Immunol. 133, 78-85 (2009).

37. Brandtzaeg, P. Mucosal immunity: integration between mother and the breast-fed infant. Vaccine 21, 3382-3388 (2003).

38. Hirose, J. et al. Occurrence of the major food allergen, ovomucoid, in human breast milk as an immune complex. Biosci. Biotechnol. Biochem. 65, 1438-1440 (2001).

39. Chirdo, F.G., Rumbo, M., Anon, M.C. \& Fossati, C.A. Presence of high levels of non-degraded gliadin in breast milk from healthy mothers. Scand. J. Gastroenterol. 33, 1186-1192 (1998).

40. Jarvinen, K.M., Laine, S.T., Jarvenpaa, A.L. \& Suomalainen, H.K. Does Iow IgA in human milk predispose the infant to development of cow's milk allergy? Pediatr. Res. 48, 457-462 (2000).

41. Savilahti, E., Siltanen, M., Kajosaari, M., Vaarala, O. \& Saarinen, K.M. IgA antibodies, TGF-beta1 and -beta2, and soluble CD14 in the colostrum and development of atopy by age 4. Pediatr. Res. 58, 1300-1305 (2005).

42. Savilahti, E. et al. Low colostral IgA associated with cow's milk allergy. Acta. Paediatr. Scand. 80, 1207-1213 (1991).

43. Mosconi, E. et al. Breast milk immune complexes are potent inducers of oral tolerance in neonates and prevent asthma development. Mucosal Immunol. (2010). E-pub ahead of print.

44. Husby, S., Oxelius, V.A., Teisner, B., Jensenius, J.C. \& Svehag, S.E. Humoral immunity to dietary antigens in healthy adults. Occurrence, isotype and lgG subclass distribution of serum antibodies to protein antigens. Int. Arch. Allergy Appl. Immunol. 77, 416-422 (1985).

45. Haddad, Z.H., Vetter, M., Friedmann, J., Sainz, C. \& Brunner, E. Detection and kinetics of antigen-specific lgE and lgG immune complexes in food allergy. Ann. Allergy 51, 255 (1983).

46. Casas, R. \& Bjorksten, B. Detection of Fel d 1-immunoglobulin G immune complexes in cord blood and sera from allergic and non-allergic mothers. Pediatr. Allergy Immunol. 12, 59-64 (2001).

47. Cianga, P., Medesan, C., Richardson, J.A., Ghetie, V. \& Ward, E.S. Identification and function of neonatal Fc receptor in mammary gland of lactating mice. Eur. J. Immunol. 29, 2515-2523 (1999).

48. Kim, Y.W. \& Halsey, J.F. Metabolism and clearance of antibody-excess immune complexes in lactating mice. J. Immunol. 129, 619-622 (1982).

49. Pacha, J. Development of intestinal transport function in mammals. Physiol. Rev. 80, 1633-1667 (2000).

50. Cummins, A.G. \& Thompson, F.M. Postnatal changes in mucosal immune response: a physiological perspective of breast feeding and weaning. Immunol. Cell Biol. 75, 419-429 (1997).

51. Macdonald, T.T. \& Monteleone, G. Immunity, inflammation, and allergy in the gut. Science 307, 1920-1925 (2005).

52. Chehade, M. \& Mayer, L. Oral tolerance and its relation to food hypersensitivities. J. Allergy Clin. Immunol. 115, 3-12; quiz 13 (2005).

53. Heyman, M., Crain-Denoyelle, A.M., Corthier, G., Morgat, J.L. \& Desjeux, J.F. Postnatal development of protein absorption in conventional and germ-free mice. Am. J. Physiol. 251, G326-G331 (1986).

54. Ménard, S., Cerf-Bensussan, N. \& Heyman, M. Multiple facets of intestinal permeability and epithelial handling of dietary antigens. Mucosal Immunol. 3, 247-259 (2010).

55. Brambell, F.W. The transmission of immunity from mother to young and the catabolism of immunoglobulins. Lancet 2, 1087-1093 (1966).

56. Ghetie, V. \& Ward, E.S. Transcytosis and catabolism of antibody. Immunol. Res. 25, 97-113 (2002). 
57. Roopenian, D.C. \& Akilesh, S. FcRn: the neonatal Fc receptor comes of age. Nat. Rev. Immunol. 7, 715-725 (2007).

58. Qiao, S.W., Lencer, W.I. \& Blumberg, R.S. How the controller is controlled-neonatal Fc receptor expression and immunoglobulin $\mathrm{G}$ homeostasis. Immunology 120, 145-147 (2007).

59. Abrahamson, D.R., Powers, A. \& Rodewald, R. Intestinal absorption of immune complexes by neonatal rats: a route of antigen transfer from mother to young. Science 206, 567-569 (1979).

60. Yoshida, M. et al. Human neonatal Fc receptor mediates transport of lgG into luminal secretions for delivery of antigens to mucosal dendritic cells. Immunity 20, 769-783 (2004).

61. Yoshida, M. et al. Neonatal Fc receptor for lgG regulates mucosal immune responses to luminal bacteria. J. Clin. Invest. 116, 2142-2151 (2006).

62. Kelsall, B. Recent progress in understanding the phenotype and function of intestinal dendritic cells and macrophages. Mucos. Immunol. 1, 460-469 (2008).

63. Macpherson, A.J. \& Smith, K. Mesenteric lymph nodes at the center of immune anatomy. J. Exp. Med. 203, 497-500 (2006).

64. Randall, T.D., Carragher, D.M. \& Rangel-Moreno, J. Development of secondary lymphoid organs. Annu. Rev. Immunol. 26, 627-650 (2008).

65. Coombes, J.L. \& Powrie, F. Dendritic cells in intestinal immune regulation. Nat. Rev. Immunol. 8, 435-446 (2008).

66. Strober, W. Vitamin A rewrites the ABCs of oral tolerance. Mucos. Immunol. 1, 92-95 (2008).

67. Adkins, B., Leclerc, C. \& Marshall-Clarke, S. Neonatal adaptive immunity comes of age. Nat. Rev. Immunol. 4, 553-564 (2004).

68. Mayrhofer, G., Pugh, C.W. \& Barclay, A.N. The distribution, ontogeny and origin in the rat of la-positive cells with dendritic morphology and of la antigen in epithelia, with special reference to the intestine. Eur. J. Immunol. 13, 112-122 (1983).

69. van Rees, E.P., Dijkstra, C.D., van der Ende, M.B., Janse, E.M. \& Sminia, T. The ontogenetic development of macrophage subpopulations and la-positive non-lymphoid cells in gut-associated lymphoid tissue of the rat. Immunology 63, 79-85 (1988).

70. Billingham, R.E. \& Brent, L. Acquired tolerance of foreign cells in newborn animals. Proc. R. Soc. London B Biol. Sci. 146, 78-90 (1956).

71. Brent, L. The 50th anniversary of the discovery of immunologic tolerance. N. Engl. J. Med. 349, 1381-1383 (2003).

72. Billingham, R.E., Brent, L. \& Medawar, P.B. Actively acquired tolerance of foreign cells. Nature 172, 603-606 (1953).

73. Sarzotti, M., Robbins, D.S. \& Hoffman, P.M. Induction of protective CTL responses in newborn mice by a murine retrovirus. Science $\mathbf{2 7 1}$, 1726-1728 (1996)

74. Ridge, J.P., Fuchs, E.J. \& Matzinger, P. Neonatal tolerance revisited: turning on newborn T cells with dendritic cells. Science 271, 1723-1726 (1996).

75. Forsthuber, T., Yip, H.C. \& Lehmann, P.V. Induction of TH1 and TH2 immunity in neonatal mice. Science 271, 1728-1730 (1996).

76. Karlsson, M.R., Rugtveit, J. \& Brandtzaeg, P. Allergen-responsive $\mathrm{CD} 4+\mathrm{CD} 25+$ regulatory $T$ cells in children who have outgrown cow's milk allergy. J. Exp. Med. 199, 1679-1688 (2004).

77. Lack, G. Epidemiologic risks for food allergy. J. Allergy Clin. Immunol. 121, 1331-1336 (2008).

78. Lamont, A.G., Mowat, A.M., Browning, M.J. \& Parrott, D.M. Genetic control of oral tolerance to ovalbumin in mice. Immunology 63, 737-739 (1988).

79. Vercelli, D. Discovering susceptibility genes for asthma and allergy. Nat. Rev. Immunol. 8, 169-182 (2008).

80. Gross, L. Microbes colonize a baby's gut with distinction. PLoS Biol. 5, e191 (2007).

81. Palmer, C., Bik, E.M., DiGiulio, D.B., Relman, D.A. \& Brown, P.O. Development of the human infant intestinal microbiota. PLOS Biol. 5, e177 (2007).

82. Bjorksten, B. Effects of intestinal microflora and the environment on the development of asthma and allergy. Springer Semin. Immunopathol. 25, 257-270 (2004).

83. Macpherson, A.J. \& Harris, N.L. Interactions between commensal intestinal bacteria and the immune system. Nat. Rev. Immunol. 4, 478-485 (2004).

84. Round, J.L. \& Mazmanian, S.K. The gut microbiota shapes intestinal immune responses during health and disease. Nat. Rev. Immunol. 9, 313-323 (2009).

85. Mazmanian, S.K., Liu, C.H., Tzianabos, A.O. \& Kasper, D.L. An immunomodulatory molecule of symbiotic bacteria directs maturation of the host immune system. Cell 122, 107-118 (2005).
86. Gaboriau-Routhiau, V. et al. The key role of segmented filamentous bacteria in the coordinated maturation of gut helper $\mathrm{T}$ cell responses. Immunity 31, 677-689 (2009).

87. Mazmanian, S.K. Gut immune balance is as easy as S-F-B. Immunity 31, 536-538 (2009).

88. Wen, L. et al. Innate immunity and intestinal microbiota in the development of type 1 diabetes. Nature 455, 1109-1113 (2008).

89. Bach, J.F. The effect of infections on susceptibility to autoimmune and allergic diseases. N. Engl. J. Med. 347, 911-920 (2002).

90. Perez, P.F. et al. Bacterial imprinting of the neonatal immune system: lessons from maternal cells? Pediatrics 119, e724-e732 (2007).

91. Menezes, J.S. et al. Stimulation by food proteins plays a critical role in the maturation of the immune system. Int. Immunol. 15, 447-455 (2003).

92. Mucida, D.S. et al. Decreased nasal tolerance to allergic asthma in mice fed an amino acid-based protein-free diet. Ann. NY Acad. Sci. 1029, 361-365 (2004).

93. Maynard, C.L. et al. Regulatory T cells expressing interleukin 10 develop from Foxp3(+) and Foxp3(-) precursor cells in the absence of interleukin 10. Nat. Immunol. 8, 931-941 (2007).

94. Penttila, I.A. et al. Transforming growth factor-beta levels in maternal milk and expression in postnatal rat duodenum and ileum. Pediatr. Res. 44, 524-531 (1998).

95. Letterio, J.J. et al. Maternal rescue of transforming growth factor-beta 1 null mice. Science 264, 1936-1938 (1994).

96. Penttila, I.A. et al. Maternal milk regulation of cell infiltration and interleukin 18 in the intestine of suckling rat pups. Gut 52, 1579-1586 (2003).

97. Penttila, I. Effects of transforming growth factor-beta and formula feeding on systemic immune responses to dietary beta-lactoglobulin in allergyprone rats. Pediatr. Res. 59, 650-655 (2006).

98. Cummins, A.G. \& Thompson, F.M. Effect of breast milk and weaning on epithelial growth of the small intestine in humans. Gut 51, 748-754 (2002).

99. Oddy, W.H. et al. TGF-beta in human milk is associated with wheeze in infancy. J. Allergy Clin. Immunol. 112, 723-728 (2003).

100. Bouaziz, J.D., Yanaba, K. \& Tedder, T.F. Regulatory B cells as inhibitors of immune responses and inflammation. Immunol. Rev. 224, 201-214 (2008).

101. Daulaire, N.M. et al. Childhood mortality after a high dose of vitamin A in a high risk population. BMJ 304, 207-210 (1992).

102. Rahmathullah, L. et al. Reduced mortality among children in southern India receiving a small weekly dose of vitamin A. N. Engl. J. Med. 323, 929-935 (1990).

103. Benjamin, D.C. Neonatally induced tolerance to HGG: duration in B cells and absence of specific suppressor cells. J. Immunol. 119, 311-314 (1977).

104. Sicherer, S.H. The impact of maternal diets during breastfeeding on the prevention of food allergy. Curr. Opin. Allergy Clin. Immunol. 2, 207-210 (2002).

105. Greer, F.R., Sicherer, S.H. \& Burks, A.W. Effects of early nutritional interventions on the development of atopic disease in infants and children: the role of maternal dietary restriction, breastfeeding, timing of introduction of complementary foods, and hydrolyzed formulas. Pediatrics 121, 183-191 (2008).

106. Lack, G. The concept of oral tolerance induction to foods. Nestle Nutr. Workshop Ser. Pediatr. Program 59, 63-68; discussion 68-72 (2007).

107. Zeiger, R.S. Food allergen avoidance in the prevention of food allergy in infants and children. Pediatrics 111, 1662-1671 (2003).

108. Poole, J.A. et al. Timing of initial exposure to cereal grains and the risk of wheat allergy. Pediatrics 117, 2175-2182 (2006).

109. Du Toit, G. et al. Early consumption of peanuts in infancy is associated with a low prevalence of peanut allergy. J. Allergy Clin. Immunol. 122, 984-991 (2008).

110. Host, A. Frequency of cow's milk allergy in childhood. Ann. Allergy Asthma Immunol. 89, 33-37 (2002).

111. van Odijk, J. et al. Breastfeeding and allergic disease: a multidisciplinary review of the literature (1966-2001) on the mode of early feeding in infancy and its impact on later atopic manifestations. Allergy 58, 833-843 (2003).

112. Gdalevich, M., Mimouni, D. \& Mimouni, M. Breast-feeding and the risk of bronchial asthma in childhood: a systematic review with meta-analysis of prospective studies. J. Pediatr. 139, 261-266 (2001). 